\title{
More Evidence for Repeat PAE and Different Patterns of Vascular Reperfusion of the Prostate
}

\author{
Iñigo Insausti ${ }^{1} \cdot$ Antonio Martinez $^{1}$
}

Received: 4 November 2020/ Accepted: 25 November 2020/Published online: 3 January 2021

(C) Springer Science+Business Media, LLC, part of Springer Nature and the Cardiovascular and Interventional Radiological Society of Europe (CIRSE) 2021

\section{Introduction}

Despite the safety and effectiveness of the prostatic artery embolisation (PAE), the percentage of patients with clinical failure following the procedure ranges between 3 and $30 \%$ according to different studies [1]. At present, the reason for this is unclear and it is thought that prostatic revascularisation may play a role.

Recently, Kearns et al. have published in this same journal an article under the title "Patterns of Reperfusion and Clinical Findings in Repeat Prostate Artery Embolisation for Recurrent Lower Urinary Tract Symptoms in Patients with Benign Prostatic Hyperplasia" [2].

This is a single-centre, nonrandomised, retrospective study looking into the reperfusion pattern and the clinical results at 3 months in 12 patients undergoing a repeat PAE (rPAE). The patients included in the study had a good clinical response after PAE, with an IPSS (International Prostate Symptom Score) decrease greater than 25\% followed by recurrence after a latency period. Patients not achieving clinical success after PAE were excluded from the study.

Following rPAE patients experienced 9.9-point reduction in IPSS $(p<0.0001)$ and 2-point reduction in quality of life $(\mathrm{QoL})(p=0.0002)$. There was a clinical success rate of $66 \%$ with no significant difference between unilateral and bilateral PAE (52.3 vs $54.3 \%, p=0.55$ ). In addition, predominant pattern for revascularisation was through recanalisation of the main prostatic artery in $80 \%$ of the hemiprostates and through other pelvic arterial branches (internal pudendal, obturator, posterolateral prostatic, etc.) in $20 \%$.

This study reinforces the previous evidence on the effectivity of rPAE in patients with recurring lower urinary tract symptoms (LUTS) [3, 4]. The results after rPAE in the study population are similar to those after initial PAE and to those published by other authors, with IPSS and QoL improvement of 9.5 and 1.3 points, respectively [4].

Regarding patient selection, the authors suggest that rPAE should be reserved for that group of patients presenting a relapse of symptoms after an initial satisfactory clinical response after PAE (relapsers). Likewise, they propose that rPAE should be avoided in those patients not experiencing clinical improvement after PAE (nonresponders). This is in accordance with the results published by Costa et al. [4], who found clinical response at 12 months in $57 \%$ of relapsers, but only of $28 \%$ in nonresponders.

In connection with the pattern of prostatic revascularisation, the study describes most of these being dependent on the main prostate artery, much in agreement with Costa et al. findings, which found the same pattern in 76 out of 108 patients analysed [4]. These observations suggest that

\footnotetext{
Iñigo Insausti

iinsausti@unav.es; insausti00@hotmail.com

Antonio Martinez amartinc@unav.es

1 Vascular and Interventional Radiology Department, Clinica Universidad de Navarra, Pamplona, Navarra, Spain
} 
there might be a need for technical improvements in order to reduce the rate of recanalisation following PAE and therefore the number of patients with clinical relapse of symptoms. The suggestion is made to embolise in a more extensive and definitive manner the main prostate artery by using coils or liquid embolic agents, the drawback of this being that these may add to the technical difficulty if an rPAE becomes necessary. Other authors have proposed more aggressive embolisation by the use of smaller particles [5] or techniques allowing for a more distal embolisation [6].

In conclusion, we believe that this article demonstrates the efficacy of rPAE in patients who have shown initial clinical improvement followed by true recurrence of symptoms after PAE, and raises the possible need for a more aggressive initial PAE in order to decrease the rate of recanalisation of the main prostate artery and therefore the clinical failure rate.

Funding Not applicable.

\section{Compliance with Ethical Standards}

Conflict of interest The authors have no conflict of interest.

Ethical Approval Not applicable.

Informed Consent Not applicable.

Consent for Publication Not applicable.

\section{References}

1. Bilhim T, Pisco J, Pinheiro LC, et al. Does polyvinyl alcohol particle size change the outcome of prostatic arterial embolization for benign prostatic hyperplasia? Results from a single-center randomized prospective study. J Vasc Interv Radiol. 2013;24:1595-602.

2. Kearns DJ, Boardman P, Tapping CR. Patterns of reperfusion and clinical findings in repeat prostate artery embolisation for recurrent lower urinary tract symptoms in patients with benign prostatic hyperplasia. Cardiovasc Intervent Radiol. 2020. https://doi.org/10. 1007/s00270-020-02671-7 (PMID: 33037455).

3. Pisco JM, Bilhim T, Pinheiro LC, et al. Medium- and long-term outcome of prostate artery embolization for patients with benign prostatic hyperplasia: results in 630 patients. J Vasc Interv Radiol. 2016;27(8):1155-1122.

4. Costa NV, Torres D, Pisco J, Pinheiro LC, Martins FE, Oliveira AG, Bilhim T. Repeat prostatic artery embolization for patients with benign prostatic hyperplasia. $J$ Vasc Interv Radiol. 2020;31(8):1272-80. https://doi.org/10.1016/j.jvir.2020.04.027 (PMID: 32741552).

5. Wang MQ, Zhang JL, Xin HN, et al. Comparison of clinical outcomes of prostatic artery embolization with 50- $\mu \mathrm{m}$ Plus $100-\mu \mathrm{m}$ polyvinyl alcohol (PVA) particles versus $100-\mu \mathrm{m}$ PVA particles alone: a prospective randomized trial. J Vasc Interv Radiol. 2018;29(12):1694-702.

6. Carnevale FC, Moreira AM, Antunes AA. The "PErFecTED technique": proximal embolization first, then embolize distal for benign prostatic hyperplasia. Cardiovasc Intervent Radiol. 2014;37(6):1602-5.

Publisher's Note Springer Nature remains neutral with regard to jurisdictional claims in published maps and institutional affiliations. 